\title{
Forschungsdatenmanagement und Bibliotheken
}

\author{
Silke Bellanger*, Gérard Bagnoud**, Eliane Blumer***, Carmen Jambé**, Simone Rosenkranz ${ }^{a}$, \\ René Schneider ${ }^{b}$, Florian Steurer ${ }^{c}$, Stefanie Strebel ${ }^{c}, C$ Clemens Trautwein ${ }^{d}$, Matthias Töwe $^{e}$
}

\section{Zusammenfassung}

In den letzten 10 Jahren haben die meisten wissenschaftlichen Bibliotheken das Thema Forschungsdatenmanagement (FDM) aufgegriffen. Im Kontext ihrer Open Science Aktivitäten planten viele bereits früh, Services nicht nur für die freie Publikation von Forschungsartikeln zu bieten, sondern auch Angebote für die Veröffentlichung weiterer Forschungsergebnisse wie Forschungsdaten zu schaffen. Mittlerweile sind international und national die Vorhaben zu Dienstleistungen geworden, doch wie das Thema Forschungsdaten an sich, sind auch die Services der Bibliotheken weiterhin in Entwicklung und die jeweiligen Profile stark von den lokalen institutionellen und fachspezifischen Gegebenheiten geprägt.

Der in kurzer Zeit zusammengestellte Artikel bietet einen kursorischen Überblick über Forschungsdatenmanagement sowie die Angebote von Schweizer Bibliotheken in diesem Zusammenhang. Das Kollektiv der Autoren und Autorinnen stellt anhand ihrer jeweiligen Bibliotheken vor, mit welchen Dienstleistungen Bibliotheken Forschende beim Forschungsdatenmanagement unterstützen. Ähnliche oder weitergehende Angebote bieten auch die Teams der weiteren Schweizer Bibliotheken an, die aus zeitlichen Gründen nicht bei der Zusammenstellung dabei sein konnten.

\section{Forschungsdatenmanagement - was ist das?}

Die Europäische Kommission definiert Forschungsdaten als: «Dokumente in digitaler Form, bei denen es sich nicht um wissenschaftliche Veröffentlichungen handelt und die im Laufe von wissenschaftlichen Forschungstätigkeiten erfasst oder erzeugt und als Nachweise im Rahmen des Forschungsprozesses verwendet werden oder die in der Forschungsgemeinschaft allgemein für die Validierung von Forschungsfeststellungen und -ergebnissen als notwendig erachtet werden ${ }^{1}$. Dabei kann es sich um Statistiken, Versuchsergebnisse, Messungen, Beobachtungen aus der Feldarbeit, Umfrageergebnisse, Befragungsaufzeichnungen und Bilder oder Audio handeln. Auch Metadaten, Spezifikationen und andere digitale Objekte wie Software sind Teil davon. ${ }^{2}$

In den letzten Jahren ist die Verfügbarkeit und die Weiterverwendbarkeit eben dieser Forschungsdaten,

\footnotetext{
1 https://eur-lex.europa.eu/legal-content/DE/TXT/?uri=CELEX:32019L1024, Artikel 2, Punkt 9

2 https://eur-lex.europa.eu/legal-content/DE/TXT/?uri=CELEX:32019L1024, Richtlinie, Punkt 27
}

welche zum grössten Teil aus öffentlichen Mitteln finanziert werden, Gegenstand unterschiedlichster politischer Initiativen geworden. Die Hauptziele sind ein möglichst schneller, offener und digitaler Zugang und die kostenlose und uneingeschränkte Ver-

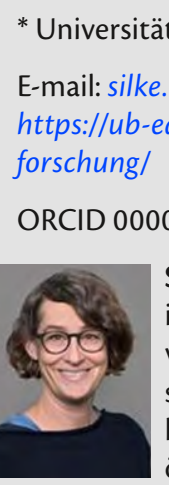

ersitätsbibliothek Basel, Schönbeinstrasse 18-20, 4056 Basel.

E-mail: silke.bellanger@unibas.ch

https://ub-easyweb.ub.unibas.ch/de/open-science-publizieren-digitaleforschung/

ORCID 0000-0003-1952-1933 Informations- und Bibliothekswissenschaften. Sie ist aktuell Mitglied des Schweizerischen Arbeitskreises Open Access und der Arbeitsgruppe für eine nationale Open Research Data Strategy.

** Université de Lausanne, UNIRIS - Ressources Informationnelles et archives, Quartier UNIL-Centre, Bâtiment Unicentre, 1015 Lausanne.

E-mail: gerard.bagnoud@unil.ch,carmen.jambe@unil.ch www.unil.ch/uniris

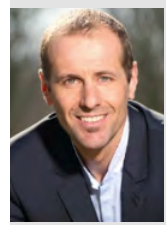

Gérard Bagnoud, Diplômé de l'Institut de Hautes Etudes Internationales et du Développement, a suivi de nombreuses formations certifiantes en archivistique (de l'Association des archivistes suisses), en gestion électronique de documents et en Electronic Records Management (certification AlIM). II a notamment travaillé aux Archives d'Etat de Genève, pour l'entreprise horlogère Rolex ainsi qu'au Pouvoir judiciaire genevois. Il est actuellement directeur du service des Ressources informationnelles et archives (UNIRIS) de l'UNIL depuis 2013. Au sein d'UNIRIS, on développe depuis 2015 un secteur d'activité autour de la gestion des données de la recherche et de l'Open Science. En parallèle, Gérard Bagnoud est professeur invité à la Haute Ecole de Gestion de Genève, filière information documentaire, et membre du Comité scientifique de la HES-SO en science de l'information. Il est également membre du Conseil consultatif du programme de formation continue en archivistique, bibliothéconomie et sciences de l'information des universités de Lausanne et de Berne. Als Absolvent des Graduate Institute of International and Development Studies hat Gérard Bagnoud zahlreiche Zertifizierungskurse in den Bereichen Archivierung (vom Verband Schweizerischer Archivare), elektronische Dokumentenverwaltung und Electronic Records Management (AllM-Zertifizierung) absolviert. Er hat insbesondere im Genfer Staatsarchiv, für die Uhrenfirma Rolex und für die Genfer Justiz gearbeitet. Derzeit und seit 2013 ist er Direktor des Dienstes für Informationsressourcen und Archive (UNIRIS) an der UNIL. Innerhalb von UNIRIS wurde seit 2015 ein Aktivitätsbereich rund um Forschungsdatenmanagement und Open Science entwickelt. Gleichzeitig ist Bagnoud Gastprofessor an der Hochschule für Wirtschaft Genf (HES-SO) im Bereich Dokumentarinformation und Mitglied des wissenschaftlichen Ausschusses der HES-SO für Informationswissenschaft. Er ist auch Mitglied des Beirats des Weiterbildungsprogramms in Archiv-, Bibliotheks- und Informationswissenschaft der Universitäten Lausanne und Bern. https://ch.linkedin.com/in/gerardbagnoud 
Carmen Jambé, Bachelor of Science HES-SO en Information documentaire, diplômée en 2015 de la Haute école de gestion de Genève. Sur mandat du Service des ressources informationnelles et archives (UNIRIS) de l'Université de Lausanne (UNIL) et de son responsable M. Gérard Bagnoud, elle a réalisé le travail «La gestion des données de recherche à l'Université de Lausanne : enjeux transdisciplinaires». Suite à ce travail, elle a été engagée au sein du Service UNIRIS afin de développer le pôle Gestion des données de recherche, secteur dont elle est responsable. Depuis 2019 et suite à l'adoption par la Direction de l'UNIL de la Stratégie Open Science et plan d'actions 2019-2021, ce secteur, incluant notamment la thématique de l'Open research Data est intégré à l'Open Science Team de l'UNIL. Ses activités s'inscrivent dans les cinq axes prioritaires définis pour soutenir la communauté des chercheur-e-s de I'UNIL, fournissant ainsi conseil et soutien durant l'entier du projet de recherche et cycle de vie des données.

Bachelor of Science HES-SO in Dokumentarischer Information, Abschluss im Jahr 2015 an der Hochschule für Wirtschaft Genf. Im Auftrag des Service des ressources informationnelles et archives (UNIRIS) der Universität Lausanne (UNIL) und seines Leiters, Herrn Gérard Bagnoud, hat sie die Arbeit «La gestion des données de recherche à l'Université de Lausanne: enjeux transdisciplinaires» (Verwaltung der Forschungsdaten an der Universität Lausanne: transdisziplinäre Fragen) durchgeführt. Im Anschluss an diese Arbeit wurde sie innerhalb des UNIRIS-Dienstes eingestellt, um die Einheit für die Verwaltung von Forschungsdaten zu entwickeln, ein Bereich, für den sie verantwortlich ist. Seit 2019 und nach der Verabschiedung der Offenen Wissenschaftsstrategie und des Aktionsplans 2019-2021 durch das UNIL-Management ist dieser Bereich, insbesondere auch das Thema Offene Forschungsdaten, in das Offene Wissenschaftsteam der UNIL integriert. Seine Aktivitäten stehen im Einklang mit den fünf Prioritätsachsen, die zur Unterstützung der UNIL-Forschungsgemeinschaft definiert wurden, und bieten Beratung und Unterstützung während des gesamten Forschungsprojekts und Datenlebenszyklus.

*** Bibliothèque de l'Ecole Polytechnique Fédérale Lausanne, Rolex Learning Center, Station 20, 1015 Lausanne.

E-mail:eliane.blumer@epfl.ch

https://www.epfl.ch/campus/library/services/services-researchers/rdmquick-start/

\section{ORCID 0000-0002-0972-5396}

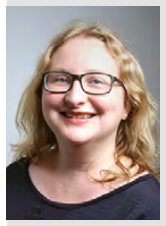

Eliane Blumer, B. Sc. und M. Sc., ist seit 2017 Koordinatorin Forschungsdatenmanagement und Fachreferentin Life Sciences an der Bibliothek der EPFL Lausanne. Nach einem Bachelor in Informationswissenschaften und einem Master in Informationssystemen arbeitete sie in unterschiedlichsten Bibliotheken.

wendung der Daten. Die Vorteile davon sind eine verbesserte Qualität, mehr Transparenz und Effizienz in der Forschung. Für Forschende kann die Veröffentlichung der Daten zudem zusätzlich die Verbreitung und Wertschätzung ihrer Arbeit fördern.

Damit die Daten jedoch auch für andere Personen als den ursprünglichen Forschenden wiederverwendbar sind, müssen sie ein gewisses Mass an Qualität und Verständlichkeit vorweisen. Aus diesem Grund werden wissenschaftspolitisch und institutionell Bemühungen unternommen, dass Forschende bereits vor und während ihres Forschungsprojekts mittels spezifischer Datenmanagementpläne überlegen, was mit ihren Daten passieren soll. Sie definieren dabei die Erhebung, die Aufbereitung und Analyse, die Interpretation und Dokumentation, die Publikation sowie die Langzeitarchivierung der Daten und halten ethische und rechtliche Probleme fest. Neben den Datenmanagementplänen unterstützen Grundsätze für gutes Forschungsdatenmanagement, wie sie z.B. die FAIR Prinzipien ${ }^{3}$ vorschlagen, die Daten so aufzubereiten, dass sie nicht nur verständlich, sondern auch für Menschen und Maschinen wiederverwendbar sind. So empfehlen die FAIR-Prinzipien Praktiken und Technologien, mit deren Hilfe Daten findbar, zugänglich, interoperabel und nachnutzbar gemacht werden können. Wie weiter unten deutlich wird, werden Forschenden je nach Organisation bei diesen Arbeitsschritten von unterschiedlichsten Abteilungen, wie zum Beispiel der Bibliothek, dem Forschungszentrum, der Informatik oder der Rechtsabteilung unterstützt.

\section{Forschungsdatenmanagement und Data Science}

Es gibt aktuell verschiedene Ansätze zum Umgang mit Daten - wie z.B. Big Data, Data Science oder eben Forschungsdatenmanagement. Da es nicht immer eine offiziell validierte Definition der jeweiligen Konzepte gibt, ist es schwierig, eine exakte Trennlinie zu ziehen. Etwas einfacher ist es, die nötigen Kompetenzen anzuschauen, die es jeweils braucht. «A broad range of data skills are needed across the research lifecycle. These cover data science skills to ensure research communities handle data appropriately and can exploit FAIR data resources, as well as stewardship skills to ensure data are FAIR, properly managed, preserved and shared. [...]..» ${ }^{4}$ Wie Hodson et al. (2018) beschreiben, braucht es ein breites Skill-Set, um den gesamten Data Life Cycle abzudecken. Es werden sowohl Fähigkeiten zur Organisation der Daten gebraucht als auch technische Kompetenzen benötigt, wobei z.B. letztere klar in der Informatik anzusiedeln sind, wie eine Studie von Modulhandbüchern von Neuroth et al. gezeigt hat. ${ }^{5}$ Die benötigten Kompetenzen sind im Forschungsprozess besonders im Moment der Datenanalyse eng miteinander verschränkt. Hier braucht es sowohl technische Kompetenzen für Data Processing, Datenversionierung, -visualisierung oder Data Mining als auch Fähigkeiten, die Daten gut zu organisieren und zu kuratieren.

\section{Internationale und nationale Entwicklungen}

Wer mit Daten und deren Management zu tun hat, kommt auf dem internationalen Teppich sowohl an

\footnotetext{
3 «Findable, Accessible, Interoperable and Reusable»,

siehe https://www.go-fair.org/fair-principles/

4 Hodson, Simon et al., 2018

5 Neuroth et al. p. 424, 2019
} 
der Research Data Alliance (RDA) ${ }^{6}$ sowie am Digital Curation Centre $(D C C)^{7}$ nicht vorbei.

Die RDA wurde 2013 von der Europäischen Kommission, der National Science Foundation und dem National Institute of Standards and Technology der US-Regierung sowie dem Innovationsministerium der australischen Regierung als eine von der Gemeinschaft getragene Initiative ins Leben gerufen, um die soziale und technische Infrastruktur für die offene gemeinsame Nutzung und Wiederverwendung von Daten aufzubauen. ${ }^{8}$

Das DCC ist ein von mehreren englischen Universitäten geführtes Kompetenzzentrum für die Kuratierung digitaler Informationen mit Schwerpunkt auf dem Aufbau von Fähigkeiten und Fertigkeiten für die Verwaltung von Forschungsdaten. ${ }^{9}$

Beide Organisationen bieten eine Reihe von Hilfestellungen und Empfehlungen zum offenen und FAIRen Umgang mit Daten.

Auf europäischer Ebene spielt die Europäische Kommission eine wichtige Rolle für das Forschungsdatenmanagement. Die grossen Forschungsunterstützungsprojekte «H2020»10 und «Horizon Europe»11 stützen sich auf Richtlinien, welche den öffentlichen Zugang der in den Projekten generierten Daten voraussetzen. ${ }^{12}$

Mit Blick auf wissenschaftliche Bibliotheken arbeitet eine Arbeitsgruppe des Verbandes Ligue des Bibliothèques Européennes de Recherche (LIBER) ebenfalls an Forschungsdatenmanagement-Richtlinien und steht in engem Austausch mit den bereits erwähnten Zentren und Verbänden. ${ }^{13}$

\section{Was passiert in der Schweiz?}

Seit 2017 verlangt der Schweizerische Nationalfonds (SNF) bei Projektanfragen einen Datenmanagementplan und den offenen Zugang zu Forschungsdaten, die einer Publikation zugrunde liegen. Der SNF folgt damit den europäischen Entwicklungen. Er unterstützt die Publikation von Daten mit bis zu 10 '000 CHF pro Projekt. Auch wenn der Datenma-

6 https://rd-alliance.org/

7 https://www.dcc.ac.uk/

8 https://rd-alliance.org/about-rda

9 https://www.dcc.ac.uk/about

10 https://ec.europa.eu/research/participants/docs/h2020-funding-guide/ cross-cutting-issues/open-access-dissemination_en.htm

11 https://ec.europa.eu/horizon_europe_en_investing_to_shape_our future

12 https://eur-lex.europa.eu/legal-content/DE/TXT/?uri=CELEX:32019L1024

13 https://libereurope.eu/strategy/research-infrastructures/rdm/members/
${ }^{a}$ Hochschule Luzern, Fachstelle Open Access, Leitung E-Science, Zentralund Hochschulbibliothek Luzern, Frohburgstrasse 3, 6002 Luzern

E-mail: Simone.Rosenkranz@zhbluzern.ch

https://www.unilu.ch/fakultaeten/tf/professuren/judaistik-und-theologie/ mitarbeitende/simone-rosenkranz/

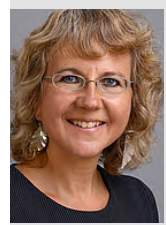

Simone Rosenkranz, Dr. phil., studierte Judaistik, Islamwissenschaften und Philosophie an den Universitäten Luzern und Basel sowie an der Hebräischen Universität Jerusalem. 1999 promovierte sie mit einer Arbeit zu den jüdisch-christlichen Beziehungen im frühislamischen Orient. Von 1999 bis 2009 war Simone Rosenkranz Assistentin am Institut für Jüdisch-Christliche Forschung der Universität Luzern. Seit 2002 ist sie zusätzlich Fachreferentin an der Zentral- und Hochschulbibliothek, wo sie zu Beginn die Fächer Judaistik und Bibelwissenschaft, seit 2005 zusätzlich die Fachbereiche Religionswissenschaften, Wissenschaftsforschung (ab 2009) und Health Sciences (ab 2013) betreut. Simone Rosenkranz hat ausserdem den Lehrauftrag für Modernhebräisch I am Institut für Jüdisch-Christliche Forschung inne.

\footnotetext{
${ }^{b}$ Haute école de gestion Genève, Rue de la Tambourine 17, 1227 Carouge.
}

E-mail: rene.schneider@hesge.ch

https://www.hesge.ch/heg/annuaire/rene-schneider/actualites

\section{ORCID 0000-0003-4897-8561}

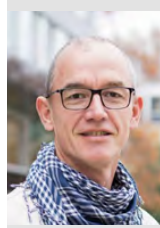

René Schneider, Dr. phil., ist Professor und Studiengangsleiter für das Fach Informationswissenschaft an der Haute Ecole de Gestion in Genf. In seiner Forschung widmet er sich den Fragen des Forschungsdatenmanagements und insbesondere der Verbindung von Linked Data und persistenter Identifikation

' Universität Zürich, Hauptbibliothek, Data Services \& Open Access, Strickhofstrasse 39, 8057 Zürich.

E-mail:data@hbz.uzh.ch

https://www.hbz.uzh.ch/de/open-access-und-open-science.html

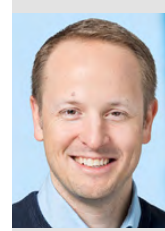

Florian Steurer, MA und Informatiker mit eidg. Fähigkeitsausweis, ist wissenschaftlicher Mitarbeiter Data Services IT \& Infrastruktur. Florian Steurer hat eine Grundbildung als Informatiker und studierte Geschichte und Lateinische Philologie an der Universität Zürich. Seit 2016 arbeitet er im Bereich Forschungsdaten im Umfeld der Universität Zürich und seit 2018 im neu etablierten Team Data Services.

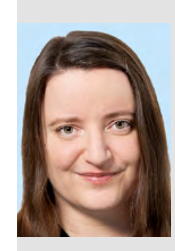

Stefanie Strebel, Dr. phil., studierte an der Universität Zürich Anglistik, Publizistik und Geographie und promovierte 2018 in Englischer Literaturwissenschaft. Sie ist seit 2019 im Team der Data Services der Universität Zürich tätig und dort für den Bereich Geistes- und Sozialwissenschaften zuständig

nagementplan (DMP) aktuell nicht Teil der Evaluation der Projektanträge ist, so muss er dennoch bei Einreichung zwingend vorhanden sein, ist während des Projektes zu aktualisieren und wird am Ende der Projektfinanzierung veröffentlicht. ${ }^{14}$ Mittlerweile sind Open Research Data, FAIR Data und Datenmanagementpläne ein Thema für alle Forschungs-

\footnotetext{
14 http://www.snf.ch/de/derSnf/forschungspolitische_positionen/open_research_data/Seiten/default.aspx
} 
${ }^{d}$ Hochschule Luzern - Technik \& Architektur,

Technikumstrasse 21, Trakt V, 6048 Horw.

E-mail: clemens.trautwein@hslu.ch

https://www.hslu.ch/en/lucerne-university-of-applied-sciences-and-arts/ about-us/people-finder/profile/?pid $=4520$

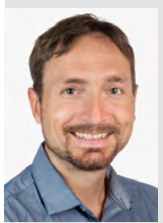

Clemens Trautwein, DI, Dr. nat. techn., erwarb 2013 an der Universität für Bodenkultur Wien das Doktorat in Umweltingenieurwissenschaften (Dr. nat. techn.), nachdem er 2007 an selbiger Universität das Diplom für Landschaftsplanung und Landschaftspflege abgeschlossen hatte. Seine fachliche Expertise vertiefte er in der Gewässerökologie und setzt nun seine Erfahrungen im analytischen Denken, Forschen und Publizieren als Fachreferent an der Hochschule Luzern, Bibliothek Technik \& Architektur ein. Er berät und schult Forschende im wissenschaftlichen Publizieren, insbesondere für Open-Access-Publikationen, sowie im Forschungsdatenmanagement.

${ }^{e}$ ETH Zürich, ETH-Bibliothek, Rämistrasse 101, 8092 Zürich.

E-mail: matthias.toewe@library.ethz.ch

https://www.library.ethz.ch/FDM

ORCID 0000-0001-9671-083X

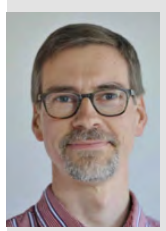

Matthias Töwe, Dr. phil. nat., leitet die Gruppe Forschungsdatenmanagement und Datenerhalt an der ETH-Bibliothek. Nach dem Studium der Chemie in Hamburg und dem Doktorat an der Universität Basel (2001) absolvierte er die Ausbildung zum Wissenschaftlichen Bibliothekar an der Universitätsbibliothek Basel. Seit 2003 arbeitet er an der ETH-Bibliothek, zunächst für das Konsortium der Schweizer Hochschulbibliotheken im Projekt E-Archiving, später als Koordinator für das Programm «Elektronische Bibliothek Schweiz - e-lib.ch».

projekte in der Schweiz. Eine Zwischenbilanz des SNF zeigt, dass etwa $70 \%$ der Datenmanagementpläne, die eingereicht wurden, gut sind und $16 \%$ das Funding in Anspruch nehmen. ${ }^{15}$

swissuniversities und seine Förderprogramme spielen ebenfalls seit mehreren Jahren eine wichtige Rolle für die FDM-Bewegung in der Schweiz. ${ }^{16}$. Einige der geförderten Projekte, wie z.B. das Projekt DLCM zu Langzeitarchivierung von Daten, ${ }^{17}$ haben die Institutionalisierung von FDM an den Hochschulen und Universitäten sowie die Zusammenarbeit zwischen den Institutionen gefördert.

Aktuell erarbeitet swissuniversities im Auftrag des SBFI eine nationale Open Research Strategie und einen dazugehörigen Aktionsplan. Komplementär zu der nationalen Open Access Strategie und dem Open Access Aktionsplan erfährt damit ein weiterer Baustein im Bereich Open Science einen national verankerten Rahmen. ${ }^{18}$

\footnotetext{
15 http://www.snf.ch/en/researchinFocus/newsroom/Pages/news200219-open-data-positive-interim-result.aspx

16 https://www.swissuniversities.ch/themen/digitalisierung/p-5-wissenschaftliche-information

17 https://dlcm.ch/

18 https://www.swissuniversities.ch/themen/digitalisierung/open-science
}

Seit 2018 besteht in der Schweiz nun ebenfalls ein nationales Forschungsdaten-Netzwerk, das sich mehrheitlich aus Mitarbeitenden der FDM-Services der wissenschaftlichen Bibliotheken zusammensetzt. Die mehr als 40 Teilnehmenden tauschen sich regelmässig aus, treffen sich zwei Mal pro Jahr und führen gemeinsame Projekte durch.

\section{Forschungsdaten und Bibliotheken}

Dienstleistungen im Bereich von Forschungsdatenmanagement schliessen an die Angebote von Bibliotheken im Bereich von Open Science an. Open Science wird zumeist definiert als «(...) the practice of science in such a way that others can collaborate and contribute, where research data, lab notes and other research processes are freely available, under terms that enable reuse, redistribution and reproduction of the research and its underlying data and methods. ${ }^{19}$ Prominent werden Open Access, Open Research Data und Open Educational Resources unter dem Umbrella-Term Open Science zusammengefasst. ${ }^{20}$ Für die Realisierung von Open Access haben viele Bibliotheken mit dem Aufbau von institutionellen Repositorien für Publikationen begonnen und ihre Dienstleistungen dann auf weitere Publikationsplattformen, Publikationsberatung und Finanzierungsmöglichkeiten ausgeweitet. Bei den Entwicklungen der Services für Forschungsdatenmanagement verhält es sich eher umgekehrt. Insbesondere aufgrund der bereits erwähnten Vorgaben der Drittmittelförderer wie dem SNF, Datenmanagementpläne mit den Forschungsanträgen einzureichen, haben die meisten Bibliotheken mit allgemeinen Beratungstätigkeiten zu Datenmanagementplänen und den verschiedenen Schritten des Lebenszyklus von Forschungsdaten begonnen.

Aktuell bieten die meisten Bibliotheken folgendes Set an Dienstleistungen an: ${ }^{21}$

- Beratungs-, Kommunikations- und Vermittlungstätigkeiten entlang des gesamten Forschungsdatenzyklus

- Publikationsinfrastrukturen und -unterstützungen

- Archivierung von Forschungsdaten

- Digitalisierung und Erschliessung von (historischen) Beständen als Datensätze

- Aufbau und Pflege von praxisbezogenen Netzwerken von Forschenden, in denen kollegial Erfahrungen und Empfehlungen weitergegeben werden.

\footnotetext{
19 Foster, n.D.

20 Für weitere Elemente von Open Science, siehe item.

21 Carol Tenopir et al. 2019. "Research Data Services in Academic Libraries: Where are We Today?" ACRL/Choice, publisher. http://choice360.org/librarianship/whitepaper.
} 


\section{Exkurs Literacies - von «Information, Media und Digital Literacy» zu «Data Literacy»}

Die Vermittlungstätigkeiten im Bereich Forschungsdatenmanagement schliessen an bereits gut etablierte Schulungs- und Kursangebote von Bibliotheken an. Wissenschaftliche Bibliotheken stützen ihre Vermittlungstätigkeit mehrheitlich auf Konzepte der «Information Literacy» ab, ein Konzept der 1990er Jahre. Die American Library Association definiert Information Literacy (IL) als «[...] a set of abilities requiring individuals to 'recognize when information is needed and have the ability to locate, evaluate, and use effectively the needed information.» 22 IL kann also als übergreifende Kompetenz aufgefasst werden, die sich auf den allgemeinen Umgang mit Informationen bezieht. Seit der Digitalisierung genügt dieser Ansatz jedoch nicht mehr. Es spielen vermehrt die «Media Literacy» - also der kritische Umgang mit nicht-textuellen Medien - sowie die «Digital Literacy» - der ethische und sichere Umgang mit Computer oder anderen technischen Ressourcen - eine nicht wegzudenkende Rolle. In den letzten Jahren hat zudem die vermehrte Zunahme von datenorientierten Aufgabenfeldern eine Notwendigkeit aufgezeigt, die IL um den Umgang mit Daten zu erweitern. Die Schlüsselkompetenz heisst «Data Literacy» und «[...] befähigt Menschen, reale Probleme durch die Nutzung, Analyse und Interpretation von Daten, die zugrunde liegenden Phänomene zu messen sowie zu adressieren.» ${ }^{23}$

Kürzlich verfasste Berichte im Bereich der digitalen Hochschulreform zeigen anschaulich auf, dass Data Literacy sehr viele Facetten hat und dass Angebote an Hochschulen oder Bibliotheken dementsprechend auch sehr unterschiedlich ausfallen können.

Der «Knowledge Synthesis Report» der Dalhousie University hat zum Beispiel fünf Hauptkompetenzbereiche entwickelt, nämlich den konzeptuellen Rahmen, Data Collection, Data Management, Data Evaluation und Data Application und verankert darin 23 Kernkompetenzen sowie 64 Hauptaufgaben. ${ }^{24}$

Im Report «Future Skills: ein Framework für Data Literacy Report» beschreiben Schüller et al. einen Kompetenzrahmen, welcher in fünf Kompetenzdimensionen aufgeteilt ist: (a) Wissen, (b) Fertigkeiten, $(c)$ Fähigkeiten, $(d)$ Motivation und $(e)$ (Wert-) Haltung. Sie sollen zur Lernzieldefinierung und zur Veranschaulichung der interdisziplinären Natur von Data Literacy beitragen. ${ }^{25}$

22 https://literacy.ala.org/information-literacy/

23 Schüller et al., 2019, p. 24

24 https://dalspace.library.dal.ca/handle/10222/64578, Appendix 1, P. 38

25 Schüller et al. 2019
Was heisst das jetzt konkret für eine wissenschaftliche Bibliothek, welche in einem grösseren Bildungskontext eingeordnet ist? Wichtig sind die Fragen: Was kann die Bibliothek alleine machen, was macht sie vielleicht schon, und wo ist institutionelle Zusammenarbeit gefragt? Die zuvor erwähnten Berichte zeigen auf, dass «Data Literacy» weit gefasst ist. Die Bibliothek hat sicher ihren Teil dazu beizutragen, ohne intensive Zusammenarbeit zwischen Bibliotheken und Nutzern ist eine sinnvolle Weiterentwicklung jedoch nicht möglich.

\section{Wie weiter?}

Der weitere Aus- und Aufbau von Dienstleistungen im Bereich FDM ist in den Bibliotheken stark abhängig von der jeweiligen Ausrichtung der Institution, den vertretenen Fachdisziplinen und der Zusammenarbeit mit weiteren Serviceprovidern, wie z.B. Informatikdiensten oder Datenzentren, lokal wie überregional. Denn zum einen können keine Service-Angebote ohne die sehr enge Zusammenarbeit mit Forschenden erfolgreich sein, aber jede Fachdisziplin hat ihr eigenes Verständnis, was Forschungsdaten sind und die Kulturen und Konventionen des Austauschs und der Veröffentlichung von Forschungsdaten sind verschieden. Zum anderen berührt das FDM viele Kompetenzen, die idealerweise ergänzend von verschiedenen Service-Stellen an Hochschulen adressiert werden. Denn FDM liegt quer zu Tätigkeits- und Zuständigkeitsbereichen wie Datenschutz, High Performance Computing, Speicherinfrastrukturen, Informationssicherheit, Publikations- und Dokumentationsinfrastrukturen, Förder- und Wissenschaftspolitik. Entsprechend werden an den meisten Hochschulen und Universitäten Services für FDM gemeinsam von Bibliotheken und Informatikdiensten angeboten. Teilweise sind dafür neue Organisationseinheiten oder Teams gebildet worden, häufig erfolgt die Arbeit aber auch in quer zu den bestehenden Einheiten organisierten Netzwerken. Und nicht zuletzt hängt der Ausbau und das Angebot eng mit den personellen Möglichkeiten der $\mathrm{Bi}$ bliotheken und weiteren beteiligten Service-Provider zusammen. Dabei ist die Anzahl an Personen genauso relevant wie das Wissen und Können der Beteiligten. Häufig werden die Aufgaben im Bereich Forschungsdatenmanagement zusätzlich zu bestehenden Funktionen übernommen und nicht immer werden neue Stellen dafür geschaffen. Zudem zeichnen sich aktuell zwar neue Berufsbilder wie «Data Librarians» $a b$, für die Ausbildungsmöglichkeiten entstehen, doch in der Regel haben gerade zu Beginn die meisten Bibliotheken und IT-Dienste parallel zu den Diensten auch die entsprechenden Kompetenzen aufgebaut. 


\section{Exkurs: Was braucht es für eine Ausbildung für zukünftige Mitarbeitende in FDM-Services in der Schweiz?}

Stellt man die Frage nach den Ausbildungen für zukünftige Mitarbeitende in FDM-Services in der Schweiz, wird schnell klar, dass sich - charakteristisch für die Schweiz - kein einheitliches, sondern ein vielstimmiges und differenziertes Bild ergibt.

Zum einen muss es Fachpersonal geben, das umfassende Kompetenzen und ein tiefes Verständnis der fachlichen Materie besitzt, im Fall des FDMs sind dies informationswissenschaftliche Kenntnisse zum Umgang mit Daten und Metadaten, insbesondere bezogen auf Repositorien oder Langzeitarchive. Diese Kompetenzen lassen sich am besten unter dem neuen Begriff der Datenkuratierung zusammenfassen und werden bereits in Ausbildungen im Bachelor- und Masterniveau der dafür vorgesehenen Fachhochschulen adressiert: zum einen für die Deutschschweiz in Chur an der FH Graubünden ${ }^{26}$, zum anderen für die Romandie an der Haute Ecole de Gestion in Genf. ${ }^{27}$

Datenkuratierung, die als Schlüsselkompetenz gilt, ist der präzise und im Hinblick auf die auszuwählenden Repositorien beziehungsweise Archive zielgenaue Umgang mit Daten und Metadaten, die auch legale und sehr datentechnische Aspekte (wie etwa Embargos, Retention Schedules, Formate, Identifikatoren, Datenmigration usw.) umfasst, letztlich Fähigkeiten, die mit der Datensicherheit zu tun haben. Zum gesamten Vorgang gehört auch die Kompetenz des Evaluierens: Welche Daten werden überhaupt erfasst und wie lässt sich deren Bedeutsamkeit abschätzen?

Das Fachpersonal muss zudem darin geschult werden, Aus-, Fort- und Weiterbildungen für verschiedene Zielgruppen geben zu können, auch für diejenigen Berufsleute, die nach Abschluss ihrer Ausbildungen in der Praxis mit FDM zu tun haben.

Weiterhin führt der Begriff FDM die Managementkompetenz ja schon mit sich. Management beweist sich in Zeiten des Wechsels und in Krisen, und «Change Management» ist angesagt, auch um Forschungsdatendesaster zu vermeiden. Allerdings können nicht alle Manager sein, sonst fehlen die Personen, die die gemanagten Prozesse ausführen (sollen).

Aufgrund des teilweise sehr weiten Zeithorizonts ist auch die Kompetenz des Planens, wie sie sich an

\footnotetext{
26 https://www.fhgr.ch/fhgr/angewandte-zukunftstechnologien/schweizerisches-institut-fuer-informationswissenschaft-sii/

27 https://www.hesge.ch/heg/en/core-programmes/bachelors-science/ information-studies
}

der konzeptionellen Wichtigkeit der Datenmanagementpläne ablesen lässt, von besonderer Wichtigkeit, so dass sich in der Summe die Kernkompetenzen der Planung, Kuratierung und Evaluierung, der Sicherung und des Managens ableiten lassen, alle im Hinblick auf Daten und Metadaten.

All dies betrifft auch den Bereich der zertifizierenden Weiterbildungsstudiengänge, genauer die CAS, DAS und MAS-Weiterbildungen. Hier gibt es bereits eine Vielzahl von Angeboten für wissenschaftliche Bibliothekare, Archivare usw.; ein spezifisches Angebot zum Bereich Datenkuratierung, das auch zukünftige Datenbibliothekare oder Datenarchivare betrifft, bleibt aber noch ein Desiderat und sollte alsbald vorzugsweise in einer konzertierten Aktion - angeboten werden, zumal der Bedarf und die Nachfrage als hoch einzuschätzen sind.

\section{Aktuelle Herausforderungen für Ausbildung, Forschende, Institutionen und Bibliotheken}

Als grösste Herausforderungen für die Ausbildung sind sicherlich fehlende Standards und die grosse $\mathrm{Di}$ vergenz zwischen generischen, $d$.h. auf alle Disziplinen und ihre Daten anwendbare Methoden und den sehr spezifischen, beinahe von Datensatz zu Datensatz oder Projekt zu Projekt, in jedem Fall aber von Disziplin zu Disziplin divergierenden Prozeduren für das Datenmanagement zu nennen. Gleichzeitig zeichnet sich derzeit ein Unterschied ab zwischen einem eher an Open Science orientierten und sehr pragmatischen Ansatz im Forschungsdatenmanagement sowie einem eher technischen und auf Langzeitarchivierung fokussierten Ansatz.

Betrachtet man die Herausforderungen aus Sicht der Forschenden und Institutionen, so üben die Forschungsförderer als externe Treiber mit zum Teil ehrgeizigen Plänen zur Umsetzung von Open Research Data und FAIR Data seit einigen Jahren erheblichen Druck auf Forschende und Institutionen aus. Insbesondere Forschende am Beginn ihrer akademischen Laufbahn finden sich in einem Spannungsfeld wieder zwischen den Anforderungen der Geldgeber, den Erwartungen ihrer Institution und den impliziten oder expliziten Zwängen für eine erfolgreiche Qualifikation in ihrem Fachgebiet. Forschenden hier eine gewisse Orientierung mit Hinweisen für praxistaugliche Lösungen zu bieten, ist eine wesentliche Herausforderung. Das Zusammenwirken unterschiedlicher Services einer Institution kann dabei ein Vorteil sein, da unterschiedliche Kompetenzen zusammenkommen. Es bedeutet aber auch Aufwand bei der Kommunikation und Abstimmung von Verantwortlichkeiten untereinander und gegenüber den Forschenden, für die die Arbeitsteilung nicht immer verständlich ist. 
In einer noch grösseren Perspektive kommt sowohl auf der Ebene der Wissenschaftspolitik als auch der Entwicklung konkreter technischer Plattformen die Herausforderung hinzu, den Anschluss an die nationalen und internationalen Entwicklungen wie die European Open Science Cloud (EOSC) ${ }^{28}$ zu behalten. Dabei ist es zumindest phasenweise gar nicht einfach herauszufinden, was die realen Ergebnisse der gross gedachten Projekte sein könnten und welche Auswirkungen diese haben könnten.

Nicht zu vernachlässigen ist auch die bereits erwähnte Schnittstelle zwischen dem FDM und den Datenwissenschaften bzw. Data Scientists. Letztere produzieren und prozessieren Daten, sie sind aber zuvorderst auf prozessierbare Daten angewiesen, wie sie das FDM liefern kann und muss.

Sollten diese Herausforderungen angenommen werden, erscheinen die Möglichkeiten nahezu unbegrenzt.

\section{Wer macht was - einige Beispiele}

Im Folgenden werden die Dienstleistungen einiger Schweizer Hochschul- und Universitätsbibliotheken vorgestellt. Ähnliche oder weitergehende Angebote bieten auch die Teams der weiteren Schweizer Bibliotheken an, die aufgrund zeitlicher Gründe nicht am Artikel mitwirken konnten. Die aufgeführten Beispiele sollten daher auch als Anregung gelesen werden, die Webseiten und Dienste der eigenen Einrichtung zu konsultieren. ${ }^{29}$

\subsection{Forschungsdatenmanagement und Service- angebote an der Hochschule Luzern (HSLU) ${ }^{30}$}

Die Bibliotheken der HSLU bieten FDM-Services seit der Einführung von obligatorischen Datenmanagementplänen bei SNF-Projektanträgen an. Die Grundlage für FDM wurde bereits 2014 im Reglement zur wissenschaftlichen Integrität für die HSLU gelegt. ${ }^{31}$ Punktuell unterstützen die zentralen IT-Services seit längerem Forschende beim Datenmanagement.

Seit 2017 arbeitet ein Team aus Fachreferenten/innen und weiteren bibliothekarischen Fachverantwortlichen zusammen, um Kompetenzen aufzubauen und FDM-Dienstleistungen anzubieten. Diese Gruppe kooperiert ausserdem mit der zentralen Ab-

\footnotetext{
28 https://ec.europa.eu/research/openscience/index.cfm?pg=open-scien ce-cloud

29 Siehe hierzu auch: https://www.forschungsdaten.org/index.php/FDMKontakte

30 https://www.hslu.ch/de-ch/technik-architektur/campus/bibliothek/ forschen-publizieren/; https://www.zhbluzern.ch/dienstleistungen/ forschen-publizieren/\#WzU2NTMsNTY1MI0=

31 Fachhochschulrat, Reglement zur wissenschaftlichen Integrität und zur guten wissenschaftlichen Praxis der Hochschule Luzern,

FH Zentralschweiz, no. 524. 2015, pp. 1-7.
}

teilung für Hochschulentwicklung im Bereich Forschung, den IT-Services und Business Applications.

Insgesamt sind etwa zehn Personen im FDM-Service mit insgesamt ein bis zwei Vollzeitäquivalenten involviert. Die Forschenden werden über interne Informationskanäle zu FDM informiert, sie können an Infoveranstaltungen der Bibliotheken teilnehmen oder sich direkt an die Bibliothek oder die IT-Services wenden.

Für Datenpublikationen in Open Access steht das institutionelle Repositorium LORY - basierend auf Zenodo - zur Verfügung. Seitens IT wird hochmoderne Infrastruktur (z.B. HPC oder Cloud Computing) zur Verfügung gestellt. Testweise wurden auch andere Anwendungen eingerichtet (z.B. Lab Notebooks).

Der Ruf nach einem institutionellen FDM-Service wird immer grösser. Deswegen läuft an der HSLU ein internes Projekt zur Realisierung eines FDM Tools, das die Forschenden in der Organisation im Datenlebenszyklus unterstützt. Der Schwerpunkt liegt dabei auf der Evaluation des Data Science Tools RENKU ${ }^{32}$ und der Verankerung des FDM Service Netzwerks zwischen den beteiligten Stellen innerhalb der Hochschule.

Die Heterogenität in der Forschung an der HSLU ist gross. Die FDM-Services müssen daher Basisbedürfnisse abdecken und auf Spezifika eingehen können. Deswegen ist eine «one-size-fits-all»-Lösung wahrscheinlich sehr schwierig umzusetzen. Die sich momentan im Aufbau befindlichen Lösungen auf nationaler und internationaler Ebene beobachten wir daher sehr genau. Der Aufbau eines zentralen FDM-Services ist eine Chance, die Kommunikation zwischen den involvierten Stellen und den Forschenden zu stärken.

\subsection{ETH Zürich ${ }^{33}$}

An der ETH Zürich sind die ETH-Bibliothek (Gruppe Forschungsdatenmanagement und Datenerhalt, FDD) und die Scientific IT Services (SIS) Träger der FDM-Unterstützung (siehe auch den Beitrag von Barillari et al. in diesem Heft). Beide Abteilungen haben sich gewissermassen von entgegengesetzten Enden her des Themas FDM angenommen.

An der ETH-Bibliothek wurde ein durchdachtes Datenmanagement spätestens im Laufe des Aufbaus eines digitalen Langzeitarchivs ab 2011 zunehmend als notwendige Voraussetzung für die spätere Publikation und Nachnutzbarkeit von Forschungsdaten erkannt. Der Aufbau von Dienstleistungen erfolgte schritt-

\footnotetext{
32 «RENKU | SDSC.» [Online]. Available:

https://datascience.ch/renku/. [Accessed: 24-Jul-2020].

33 https://ethz.ch/services/en/service/a-to-z/research-data.html
} 
weise, bis erstmals 2015 eine Mitarbeiterin mit wissenschaftlichem Hintergrund ausschliesslich für die Unterstützung des Forschungsdatenmanagements eingestellt wurde. Als wichtigstes Werkzeug wurde 2017 die Research Collection als Repositorium für Veröffentlichungen und Forschungsdaten in Betrieb genommen (https://www.research-collection.ethz.ch).

Die Sektion SIS hat ihre Wurzeln im Informatik-Support des Departements Biosystems Science and Engineering (D-BSSE) mit entsprechend hohen Anforderungen hinsichtlich Datenmengen und der Handhabung der zahlreichen Abhängigkeiten zwischen den im laufenden Forschungsprozess eingesetzten Komponenten. Mit der Datenmanagementplattform openBIS entwickelte die damalige Gruppe ab 2007 eine leistungsfähige Umgebung primär für quantitativ arbeitende Forschende. Auf der Software basieren heute mehrere Services mit unterschiedlicher Ausprägung. ${ }^{34}$

In der ETH-Bibliothek sind aktuell etwa 2.5 Stellen der Unterstützung des FDMs gewidmet, hinzu kommen Stellenanteile aus anderen Teams, vorrangig aus der Gruppe E-Publishing, welche die Research Collection betreibt und die Publikationsunterstützung leistet. Ebenso bieten Kollegen, die hauptsächlich mit der Betreuung des ETH Data Archive beschäftigt sind, Unterstützung z.B. bei Fragen der Langzeitarchivierung oder der Wahl von Dateiformaten. Bei Bedarf werden weitere Expertinnen beigezogen, etwa vom Hochschularchiv in der Bibliothek, z.B. für Fragen des Records Management, oder aus den Fachreferaten, z.B. zur Einschätzung von externen Repositorien aus fachlicher Sicht. Ausserhalb der Bibliothek sind der Rechtsdienst der ETH Zürich sowie die Technologietransferstelle ETH transfer immer wieder gefragt für die Einschätzung konkreter Fragen. Nicht zu vergessen sind die in den Departementen angesiedelten Informatik-Support-Gruppen (ISG) als Ansprechpersonen.

Die mit dem Forschungsdatenmanagement befassten Personen spielen somit in vielen Fällen auch eine wichtige Rolle als Vermittler und Lotsen in einem gerade für junge Forschende unübersichtlichen Umfeld und können so den Weg zu relevanten Informationen nicht selten stark abkürzen. Gleichzeitig gewinnen sie Schritt für Schritt selbst Know-how dazu. Sichtbar wird die breite Zusammenarbeit auch daran, dass alle genannten Stellen aktiv zum Programm der ETH RDM Summer School für junge Forschende des ETH-Bereichs beitragen.

Selbstverständlich ist die Einbindung so vieler Stellen nicht ganz einfach. Aus Sicht der ETH-Bibliothek er-

34 https://sis.id.ethz.ch/services/rdm/ öffnen diese vielfältigen Kontakte aber weitere Perspektiven auf neue Formen und Wege der Zusammenarbeit mit Partnern innerhalb und ausserhalb der Hochschule. In diesem Sinn kann FDM auch für die Bibliothek als Institution eine Möglichkeit zur Weiterentwicklung über die naheliegenden Dienstleistungen hinaus bieten.

Neben der Teilnahme an verschiedenen Formaten von Trainings und Workshops nehmen Forschende laufend per E-Mail oder telefonisch Kontakt mit der Gruppe FDD auf. Für diese Erstkontakte sind meist die Informationen auf der Webseite ausschlaggebend, inzwischen kommt vermehrt auch Mundpropaganda hinzu, wobei die Teilnehmenden von Veranstaltungen ihrerseits als Multiplikatoren wirken. Nicht selten resultieren daraus Nachfolge-Workshops speziell für einzelne Gruppen.

An der ETH Zürich spielen Kooperationen mit Industriepartnern eine noch grössere Rolle als an anderen Universitäten. Die entsprechenden Vereinbarungen begrenzen meist die Möglichkeit, Daten aus so einer Zusammenarbeit unverzüglich oder überhaupt zugänglich zu machen. Ebenfalls noch grösser als an anderen Hochschulen ist der Anteil von Forschungsgruppen, die mit grossen Datenvolumina im mehrstelligen Terabyte-Bereich umgehen. Die damit verbundenen technischen und auch wirtschaftlichen Fragen im Hinblick auf die Möglichkeit zur Erhaltung und zur Nachnutzung sind nur teilweise gelöst. Es ist jedoch ein Missverständnis zu glauben, dass das Vorhandensein von genügend preiswertem Speicher in grossem Umfang alle Probleme lösen würde. Viele Herausforderungen des Datenmanagements müssen auch dann erkannt und bewältigt werden, wenn die Datenmengen überschaubar bleiben oder die Speicherung kein Problem darstellt.

\subsection{Das Team Data Services der Hauptbibliothek der Universität Zürich ${ }^{35}$}

Die an der Hauptbibliothek angesiedelten Data Services der Universität Zürich werden seit 2018 in enger Kooperation mit der Zentralbibliothek und der S3IT der Zentralen Informatik angeboten. Das Team besteht neben der Leitung aus drei Mitarbeitenden, die jeweils einen unterschiedlichen Schwerpunkt (Naturwissenschaften und Medizin / Geistes- und Sozialwissenschaften / IT und Infrastruktur) betreuen. Für die Forschenden beinhaltet der Service hauptsächlich individuelle Beratung, Reviews von Datenmanagementplänen, sowie Schulungen und Workshops im Bereich FDM.

\footnotetext{
35 https://www.hbz.uzh.ch/de/open-access-und-open-science.html
} 
Seit Januar 2019 ist die Universität Zürich gemeinsam mit der Universität Lausanne Partnerin von SWISSUbase. Ziel ist der Aufbau eines schweizweiten Repositories. Die Projektleitung liegt bei FORS in Lausanne und verfügt über langjährige Erfahrung im Betrieb eines Repositories für die Sozialwissenschaften. Seitens der Universität Zürich möchte man das Projekt nutzen, um linguistische Daten zu verzeichnen und nachnutzbar zu machen. Dazu wurde eine enge Zusammenarbeit mit der an der UZH angesiedelten Linguistic Research Infrastructure (LiRI) beschlossen. Schwerpunkt wie Herausforderung stellte das Erstellen eines disziplinären Metadatenschemas dar. Von Beginn weg orientierte man sich an den konkreten Bedürfnissen der linguistischen Community und diskutierte die Ergebnisse mit der Projektleitung von SWISSUbase, so dass inzwischen ein Kernmodell für linguistische Metadaten vorliegt. Ebenso arbeitete man bei der Lösungsfindung mit, wie diese Metadaten visuell angeordnet und dargestellt werden sollen. Da man die linguistischen Daten zu einem späteren Zeitpunkt im internationalen Virtual Language Observatory von CLARIN, dem europäischen Netzwerk für Linguistik, auffindbar machen möchte, stehen nun Abklärungen an, wie man ein geeignetes Metadaten-Profil für CLARIN erstellt. Gegenüber den Forschenden von LiRI nehmen die Data Services primär zwei Rollen wahr. Nebst den bereits geschilderten Tätigkeiten, bei denen es um konkrete Realisierungen wie dem Erstellen von Metadatenschemata geht, agierten sie auch im Bereich des Anforderungsmanagements. So stellte sich früh heraus, dass man seitens der Linguistic Research Infrastructure an einer API für den automatisierten, skriptgesteuerten Ingest von Daten interessiert ist.

\subsection{Das FDM-Team an der EPFL-Bibliothek in Lausanne ${ }^{36}$}

Erste Überlegungen zu einem Forschungsdatenmanagement-Service begannen 2012 in der Bibliothek, und zwei Teilzeit-Stellen unterstrichen die Wichtigkeit des Themas und verstetigten ein erstes Angebot. Im Jahr 2014 kam der Service dank des Open-Data-Pilotprojekts der Europäischen Kommission und neuer, ausschließlich FDM-bezogener Stellenbesetzungen richtig in Schwung. Der interne Start eines offiziellen FDM-Services erfolgte 2015 zusammen mit dem Research Office, dem Technology Transfer Office und den zentralen IT-Diensten. Im Jahr 2016 wurde das von swissuniversities P-5 unterstützte Projekt DLCM lanciert, welches zu einer Konsolidierung der FDMDienste in der Bibliothek führte. Nur ein Jahr später

\footnotetext{
36 https://www.epfl.ch/campus/library/services/services-researchers/ rdm-quick-start/
}

unterstrich der neue Präsident der EPFL, Martin Vetterli, die strategische Bedeutung von Open Science und unterstützte somit das bereits existierende Angebot, genauso wie die Richtlinien des SNF rund um Open Data. Zu diesem Zeitpunkt entschied sich die Bibliothek, eine komplett neue Website einzurichten, um der Nachfrage gerecht zu werden. 2018 konnten weitere zwei Stellen für Data Librarians im FDM-Service besetzt werden. Eine Data Champions Community ermöglicht seit 2019 einen noch breiteren Austausch, und das laufende Jahr konzentriert sich auf die Entwicklung der technischen Publikations- und Archivinfrastruktur.

Gegenwärtig arbeiten fünf Mitarbeitende, insgesamt 2.7. FTE im FDM-Team. Alle haben einen unterschiedlichen Hintergrund, sind entweder ehemalige Forschende oder aber Informationswissenschaftler und Informationssystemexperten. Die enge interne Zusammenarbeit erstreckt sich auf die Zentral-IT / Fakultäts-ITs, das Forschungsbüro, die Technologietransferstelle sowie das Open Science Strategic Committee auf Präsidentenebene und dessen interne Projektausschreibungen. Zudem arbeitet die Bibliothek aktuell im swissuniversities-Projekt EasyFAIR ${ }^{37}$ mit.

Das aktuelle FDM-Dienstleistungsangebot umfasst Beratung, Vermittlung und Unterstützung für den gesamten Data-Life-Cycle, einschliesslich Datenmanagementplänen, Datendokumentation und Metadaten, Datenpublikation, Lab Notebooks, Datenformate, Datenpublikation, Datenlizenzierung, Datenkuratierung und -archivierung. Alle Angebote können je nach Bedarf durch spezifische, personalisierte Schulungen auf einzelne Anfragen und Bedürfnisse oder durch die Buchung eines Datenbibliothekars ausgeführt werden. Darüber hinaus bietet die Bibliothek gut 15 regelmässige RDM-Schulungen pro Jahr an, die sich an verschiedene Zielgruppen richten (z.B. Doktoranden oder allgemeine Mitarbeiter) und unterschiedliche pädagogische Ansätze verfolgen (z.B. lange oder kurze Workshops oder Demos).

Um die Forschenden bei ihren täglichen Aufgaben zu unterstützen, wurden Datenstrategie- und DMPTemplates ausgearbeitet. Die «Data Champions Community» mit mehr als 30 Forschenden aus verschiedenen Disziplinen ${ }^{38}$ steht für den fachlichen Austausch und als "Community of Practice» zur Verfügung und wird von der Bibliothek koordiniert. Aktuell arbeitet die EPFLBibliothek mit der EPFL-IT

\footnotetext{
37 https://www.swissuniversities.ch/themen/digitalisierung/p-5-wissenschaftliche-information/projekte/swiss-mooc-service-1-1-1

38 https://www.epfl.ch/campus/library/services/services-researchers/rdm-
} contacts-communities/epfl-data-champions/ 
an der Weiterentwicklung der technischen Infrastruktur und hat sich zum Ziel gesetzt, demnächst ein Datenrepository und -archiv, als Erweiterung des Open Access Repository anzubieten.

\subsection{Netzwerk Forschungsdatenmanagement an der Universität Basel ${ }^{39}$}

An der Universität Basel bietet seit 2018 ein Netzwerk universitärer Stellen Unterstützung zu FDM an. Zu dem Netzwerk gehören u.a. sciCORE - das universitäre Zentrum für wissenschaftliche Informatik, die Informatikdienste, die Datenschutzbeauftragte, das Departement für Klinische Forschung, das Grants Office, Ressort Forschung und die Universitätsbibliothek. Das Netzwerk ist ausserdem eng verbunden mit dem Center for Data Analytics und dem Digital Humanities Lab. Insgesamt sind rund 12 Personen regelmässig mit FDM befasst. Alle übernehmen diese Aufgaben zusätzlich zu ihren bestehenden Funktionen.

Das Netzwerk pflegt die gemeinsame Informationsplattform, bietet individuelle Beratungen an und führt regelmässige Trainings und Workshops durch. Neben wiederkehrenden fachübergreifenden Angeboten werden Workshops zu speziellen Problemen durchgeführt. So wurde gemeinsam mit dem Open Science Team der Universitätsbibliothek Bern ${ }^{40}$ ein Workshop zu Datenschutz und qualitativen Forschungsdaten ${ }^{41}$ durchgeführt, in dessen Zentrum die Diskussion von Forschungsprojekten von Geistesund Sozialwissenschaftlerlnnen standen. Aufbauend auf die Ergebnisse des Workshops werden Best Practices zu Datenschutzfragen für weitere Forschende zusammengestellt.

Zu den nächsten Schritten gehört zum einen, eine Plattform für den Austausch zwischen Forschenden zu schaffen. Als Anregung dienen hier die Data Conversations der Universität Lancaster, ${ }^{42}$ bei denen Forschende ihre Fragen und Lösungen zum Forschungsdatenmanagement vorstellen. Zum anderen sollen fachspezifische Ansprechpersonen im Rahmen eines internen Weiterbildungsprogramms in ihren Kenntnissen zu FDM gestärkt werden, um forschungsnahe Hilfestellungen leisten zu können. Dabei sind die

\footnotetext{
39 https://researchdata.unibas.ch/en/home/

40 https://www.unibe.ch/universitaet/dienstleistungen/universitaetsbibliothek/service/open_science/index_ger.html

41 Iris Lindenmann (2019): Datenschutz und Anonymisierung von qualitativen Forschungsdaten. UB Basel Blog. https://blog.ub.unibas. ch/2019/06/18/datenschutz-und-anonymisierung-von-qualitativenforschungsdaten/; Bellanger, Silke, Besmer, Christina, Kaufmann, Danielle, Keller, Anna, Lindenmann, Iris, Morger, Jennifer and Schreier, Gero. 2020. Handling Sensitive Data in Social Sciences and Humanities: Researchers|' Questions, Legal and Ethical Considerations. Zenodo. doi:10.5281/zenodo.3669957.

42 https://www.lancaster.ac.uk/library/research-data-management/dataconversations/
}

Programme für Data Stewards, wie sie in den Niederlanden bereits entwickelt werden, Vorbild. ${ }^{43}$

\subsection{Forschungsdatenmanagement an der Universität Lausanne ${ }^{44}$}

Seit 2015 ist es hauptsächlich die Abteilung UNIRIS, die FDM-Dienstleistungen anbietet. Unterstützt wird sie durch ein internes Netzwerk von Forschungsberatern (Fakultäten) und zentralen Diensten. Gemeinsam versuchen die Stellen, eine den Bedürfnissen entsprechende Unterstützung über den gesamten Lebenszyklus von Forschungsdaten anzubieten. Seit 2019 und der Verabschiedung der Open Science Strategie ${ }^{45}$ und des Aktionsplans 2019-2021 der UNIL bilden UNIRIS und die Forschungsberatenden nun das Open Science Team. ${ }^{46}$ Andere interne und externe Partner bieten ebenfalls FDM-Dienstleistungen an und arbeiten mit uns zusammen:

- BiUM ${ }^{47}$,

- DARIS 48 (FORS),

- PlaTec ${ }^{49}(\mathrm{DaSCH})$,

- Rechenzentrum \& Computing and Research Support Division - DCSR; Fragen der Speicherung, Datensicherung, Datensicherheit, Forschungsinfrastrukturen, etc..$^{50}$

- PACTT UNIL-CHUV; geistiges Eigentum, Patente, Technologietransfer usw.

- Forschungsethikkommission - CER-UNIL ${ }^{51}$; Ethik, ethische Grundsätze und Regeln, Bewertung der Einhaltung von Normen, Konformitätsbescheinigungen usw.

- Juristischer Dienst; rechtliche Aspekte,

- Disziplinäre Forschungszentren, z.B. UNIL-EPFL dhCenter ${ }^{52}$ : spezifische FDM-Fragen.

Bei UNIRIS sind zwei unbefristete Stellen mit insgesamt 120\% dem FDM gewidmet. Seit Juni 2020 vervollständigt zudem eine auf ein Jahr befristete $80 \%$-Stelle das FDM-Team. Alle Fakultäten (mit Ausnahme der HEC) haben einen Forschungsberatenden, bei welchem ein kleiner Prozentsatz seines Pflichtenheftes dem FDM zur Verfügung gewidmet

\footnotetext{
43 Siehe hierzu: Salome Scholtens, Petronella Anbeek, Jasmin Böhmer, Mirjam Brullemans-Spansier, Marije van der Geest, Mijke Jetten, Celia W G van Gelder. (2019). Function and competencies matrices for three types of data stewards 'policy', 'research' and 'infrastructure' (Version Version 2.1). Zenodo. https://zenodo.org/record/3490855. 44 https://www.unil.ch/uniris/fr/home.html

45 https://www.unil.ch/openscience/files/live/sites/openscience/files/ OpenScience/Files/UNIL_Strategie_Open_Science_Plan_D_Actions_ VF_2019.pdf

46 https://www.unil.ch/openscience/home/menuinst/who-are-we.html

47 https://www.bium.ch/en/publication-open-access/data-management/

48 https://forscenter.ch/data-services/

49 https://platec.unil.ch/

50 http://unil.ch/ci/dcsr

51 https://www.unil.ch/cerunil/home.html

52 https://dhcenter-unil-epfl.com/en/
} 
ist. Was das FDM in den Fakultäten, anderen zentralen Diensten und Partnern betrifft, ist es schwierig, die Stellenprozente zu schätzen.

UNIRIS bietet den Forschenden personalisierte Beratung (one-to-one, Telefon, E-Mail), insbesondere zu DMP (z.B. DMP des SNF), Recherche, Zusammentragen und Wiederverwendung vorhandener Daten, Dokumentation und Organisation von Daten, Datenspeicherung, -sicherung und -sicherheit (allgemeine Beratung und Weiterleitung an das Datenzentrum für spezifische Anfragen), Datenschutz und -sicherheit (allgemeine Beratung und Weiterleitung an das Datenzentrum bei spezifischen Anfragen), Veröffentlichung und gemeinsame Nutzung von Daten (offene Forschungsdaten), langfristige Speicherung, Archivierung und Aufbewahrung von Daten.

Zudem werden Workshops, Schulungstage oder Sensibilisierungsschulungen zu verschiedenen FDModer OS-Themen organisiert, wie zum Beispiel Informationsveranstaltungen in den Fakultäten zum DMP des SNF oder Workshops für Doktorierende. Ein Katalog von FDM- und OS-Online-Schulungskursen wird in den kommenden Monaten veröffentlicht.
Gegenwärtig gibt es technische Infrastrukturen für High Performance Computing (HPC) und Speicherung von sensiblen und nicht sensiblen Daten, Datensicherheitslösungen, elektronisches Dokumentenmanagement (EDM) administrativer Art, SWITCH-Infrastruktur und -Tools. Lücken gibt es noch bei der Entwicklung von elektronischen Lab Notebooks, virtuellen Forschungsumgebungen sowie einem Repository für die gemeinsame Nutzung und Langzeitarchivierung von Forschungsdaten. Dies ist auch ein Grund, weshalb UNIL Partner des swissuniversities-Projekts SWISSUbase $^{53}$ ist. Diese zukünftige Archivierungsinfrastruktur und Dienste zur Speicherung, gemeinsamen Nutzung und Förderung von Daten» werden der UNIL und ihren Forschenden ein Repository zur Verfügung stellen, das den Anforderungen des SNF entspricht. Die Beteiligung von FORS ${ }^{54}$ an diesem Projekt ist ein grosser Vorteil, da FORS seit vielen Jahren ein ausgewiesener Experte für Datenmanagement und Archivierung ist.

\footnotetext{
53 https://info.swissubase.ch/fr/

54 https://forscenter.ch/
}

\section{Literatur}

Bellanger, Silke, Christina Besmer, Danielle Kaufmann, Anne Keller, Iris Lindenmann, Jennifer Morger, and Gero Schreier. 2020. Handling Sensitive Data in Social Sciences and Humanities: Researchers|' Questions, Legal and Ethical Considerations. Zenodo. doi:10.5281/ zenodo.3669957.

Coan, Matthew. 2014. “Research Guides: Information Literacy: Guide for Students: What Is Information Literacy?” 2014. https://libguides. madisoncollege.edu/c.php?g=485946\&p=3323409.

Foster. n.D. “Open Science Definition.” https://www.fosteropenscience.eu/taxonomy/term/100.

Hodson, Simon, Sarah Jones, Sandra Collins, Françoise Genova, Natalie Harrower, Leif Laaksonen, Daniel Mietchen, Rūta Petrauskaité, and Peter Wittenburg. 2018. "Turning FAIR Data into Reality: Interim Report from the European Commission Expert Group on FAIR Data," June. https://doi.org/10.5281/zenodo.1285272.

"Information Literacy." 2017. Welcome to ALA's Literacy Clearinghouse (blog). February 7, 2017. https://literacy.ala.org/information-literacy/.

Lindenmann, Iris. 2019. “Datenschutz und Anonymisierung von qualitativen Forschungsdaten.” UB Basel Blog. https://blog.ub.unibas.ch/2019/06/18/datenschutz-und-anonymisierung-von-qualitativen-forschungsdaten/

"Members of the Research Data Management Working Group." n.d. LIBER (blog). Accessed July 29, 2020. https://libereurope.eu/strategy/research-infrastructures/rdm/members/.

Neuroth, Heike, Laura Rothfritz, Vivien Petras, and Maxi Kindling. 2019. "Digitales Datenmanagement als neue Aufgabe für wissenschaftliche Bibliotheken," October. https://doi.org/10.18452/20680.

Richtlinie (EU) 2019/1024 des Europäischen Parlaments und des Rates vom 20. Juni 2019 über offene Daten und die Weiterverwendung von Informationen des öffentlichen Sektors. 2019. 172. Vol. OJ L. http://data.europa.eu/eli/dir/2019/1024/oj/deu.

Ridsdale, Chantel, James Rothwell, Michael Smit, Hossam Ali-Hassan, Michael Bliemel, Dean Irvine, Daniel Kelley, Stan Matwin, and Bradley Wuetherick. 2015. "Strategies and Best Practices for Data Literacy Education: Knowledge Synthesis Report." Report. https://doi.org/10.13140/RG.2.1.1922.5044.

Scholtens, Salome, Petronella Anbeek, Jasmin Böhmer, Mirjam Brullemans-Spansier, Marije van der Geest, Mijke Jetten, Celia W $\mathrm{G}$ van Gelder. 2019. 'Function and competencies matrices for three types of data stewards 'policy', 'research' and 'infrastructure' (Version Version 2.1). Zenodo. https://zenodo.org/record/3490855.

Schüller, Katharina, Paulina Busch, and Carina Hindinger. 2019. “Future Skills: Ein Framework Für Data Literacy.” Zenodo. https://doi.org/10.5281/zenodo.3349865.

Tenopir, Carol, Jordan Kaufmann, Robert Sandusky, and Danielle Pollock. 2019. "Research Data Services in Academic Libraries: Where are We Today?" ACRL/Choice, publisher http:/choice360.org/librarianship/whitepaper. 